\title{
REDUCING THE EFFECTS OF SPACE-VARYING WAVELENGTH-DEPENDENT SCATTERING IN MULTISPECTRAL IMAGERY
}

\author{
Mark J. Carlotto, PSR Corp., 1400 Key Blvd. Suite 700, Arlington VA 22209 (mjcarlotto@ma.psrw.com)
}

Abstract -- A new method for reducing space-varying, wavelength-dependent scattering in multispectral imagery caused by smoke and haze is described. It is intended for use in situations where atmospheric scattering affects the shorter wavelengths and varies in space. The method converts an image in which space-varying scattering is present into an image where the scattering has been equalized over the entire image so that previously developed techniques for removing constant scattering effects can be used.

\section{BACKGROUND}

Assume that the atmosphere can be modeled as a horizontally homogeneous medium, the earth is a Lambertian reflector, and atmospheric properties which vary exponentially with altitude can be assumed to be constant over the scene [1]. When the contribution of the ambient illumination (skylight) can be neglected and the scattering is constant over the image, the brightness

$$
z(i, j)=\operatorname{ar}(i, j)+b
$$

$r$ is the radiance of the surface, $a$ represents the effects of atmospheric transmittance, sensor gain, and other multiplicative factors, and $b$ represents the contribution of the path radiance. The brightness values at two or more pixels in the image together with measurements of the surface radiance at the corresponding locations on the ground can be used to solve for $a$ and $b$. Alternatively, when ground truth is not available several methods have been developed to estimate the path radiance component [24]. When the scattering is not constant a technique developed by Lavreau [5] which subtracts a portion of the fourth tasseled cap feature [6] from each band can be used. The method effectively removes space-varying haze but causes the corrected bands to become more correlated thus altering the spectral balance of the data.

\section{METHODOLOGY}

When the effects of scattering are not constant over the image, the image formation model becomes

$$
z(i, j)=a(i, j) r(i, j)+b(i, j) .
$$

Assume that it is possible to segment the image into disjoint regions within which the radiance of the surface can be assumed to be constant. Let $S_{k}$ represent the set of pixels in the $k$-th region. Within the $k$-th region the brightness is

$$
z_{k^{\prime}}=a_{k^{\prime}} r_{k}+b_{k^{\prime}}
$$

where $r_{k}$ is the surface radiance. The terms $a_{k^{\prime}}$ and $b_{k^{\prime}}$, $k^{\prime} \in S_{k}$ represent the effective variation in the atmospheric transmittance and path radiance for the pixels within the region. The mean value of the brightness in the $k$-th region is

$$
\hat{z}_{k}=E\left[z_{k^{\prime}}\right]=E\left[a_{k^{\prime}}\right] r_{k}+E\left[b_{k^{\prime}}\right]
$$

and is computed over the pixels $k^{\prime} \in S_{k}$. We assume that the pixels within each of the $K$ regions are randomly distributed throughout the image and that the variation in scattering within the image is such that the distribution of atmospheric transmittance and path radiance effects is the same in all regions. Thus $E\left[a_{k^{\prime}}\right]=\mu_{a}=a$ and $E\left[b_{k^{\prime}}\right]=\mu_{b}=b$ are the same for all of the regions and

$$
\hat{z}_{k}=a r_{k}+b
$$

Assembling the $K$ regions back into an image we have

$$
\hat{z}(i, j)=\operatorname{ar}(i, j)+b
$$

thus reducing the space-varying case (Eq. 2) to a simpler one in which the scattering can be treated as a constant over the image (Eq. 1). A key requirement is that we are able to segment the image into regions of constant surface radiance.

The dependence of scattering on wavelength is a function of the size of the particles relative to the wavelength of the radiation [7]. In multispectral imagery, haze (and smoke) tends tend to affect the shorter wavelengths (i.e., the visible bands), often having negligible effect in the IR bands. Let the multispectral image be divided into two sets of bands $Z=\{X, Y\}$ where $X$ are the bands that are affected by haze and $Y$ are the bands that are not. To be specific we shall focus on Landsat TM (although the method is applicable to other multispectral sensors as well) and assume that in a given situation the visible bands (Landsat TM bands 1-3) are affected by haze while the infrared (IR) bands (Landsat TM bands 4,5 and 7) are not. Thus $x(i, j, m)$ is the brightness at pixel $(i, j)$ in the $m-$ th visible band and $y(i, j, n)$ is the brightness at pixel $(i, j)$ in the $n$ - th IR band.

Let $X^{M}$ and $Y^{N}$ denote the corresponding $M$ - and $N$ dimensional visible and IR subspaces where $M=N=3$. A point in the IR subspace corresponds to pixels that have the same values in the IR bands. It is conjectured that pixels with the same IR values are likely to be the same surface material with the same illumination and 
topography. In the absence of scattering, each point in the IR subspace is assumed to correspond to a point in the visible subspace. This is an approximation because the visible bands provide information not contained in the IR. If we assume that the distribution of pixels with the same IR values are randomly distributed throughout the image, when scattering is present the visible band values of those pixels will be affected by varying amounts, i.e., will have higher or lower brightness values because of increased or decreased path radiance in different parts of the image. Thus in situations where space-varying scattering is present in the visible bands, points in the IR subspace will correspond to a set of points in the visible subspace.

The correspondence between the two subspaces is used to establish a functional relationship that is used, in turn, to predict the values of the visible bands (i.e., what their values would be without scattering) as a function of the IR bands. Let $\mathbf{y}_{k}=\left\{y_{k}(n)\right\}$ denote the vector corresponding to the $k$-th unique combination of values in the IR bands where $y_{k}(n)$ is the value of the $n$-th IR band for the $k$-th combination (region). Let $\left\{\mathbf{x}_{k^{\prime}}, k^{\prime} \in S_{k}\right\}$ denote the set of visible band values that occur within the $k$-th region where $x_{k^{\prime}}(m)$ is the value of pixel $k^{\prime}$ in the $m$-th band. The joint distribution

$$
P(x(m), \mathbf{y})=P(x(m), y(1) \cdots y(N))
$$

is the probability of observing a particular combination of values in the $m$-th visible band and all $N$ IR bands. For a given combination of values in the IR bands, the optimal estimate for the value of the $m$-th visible band is given by the conditional mean:

$$
\begin{aligned}
\hat{x}_{k}(m) & =E\left[x_{k^{\prime}}(m) \mid \mathbf{y}_{k}\right] \\
& =\frac{\sum_{k^{\prime} \in S_{k}} x_{k^{\prime}}(m) P\left(x(m)=x_{k^{\prime}}(m), \mathbf{y}=\mathbf{y}_{k}\right)}{P\left(\mathbf{y}=\mathbf{y}_{k}\right)}
\end{aligned}
$$

It can be shown that the conditional mean minimizes the mean-squared prediction error of $x(m)$ as a function of $\mathbf{y}$ [8]. In other words it provides the best single haze-free value to assign to the $x_{k^{\prime}}(m)$ in each region.

\section{EXPERIMENTAL RESULTS}

The method has been evaluated on several images to date. We present results from one of these images over Bitterfeld, Germany in September 1986. Figure 1 shows the original and haze-equalized band 1 images. The results have been stretched for visual presentation. Like the dehazing technique described by Lavreau [5], our method effectively removes the space-varying haze. Lavreau's dehazing algorithm subtracts a portion of the fourth tasseled cap feature from the visible bands. As noted by Crist et al
[9] the fourth tasseled cap feature responds to atmospheric haze as well as to senescent vegetation, man-made materials (e.g., roads), some soils and water. Thus by subtracting a portion of the fourth tasseled cap feature from the visible bands, information about the surface may be lost in the process of removing the haze. One such example is shown in Figure 2 in which road information lost by de-hazing has been retained by haze equalization.
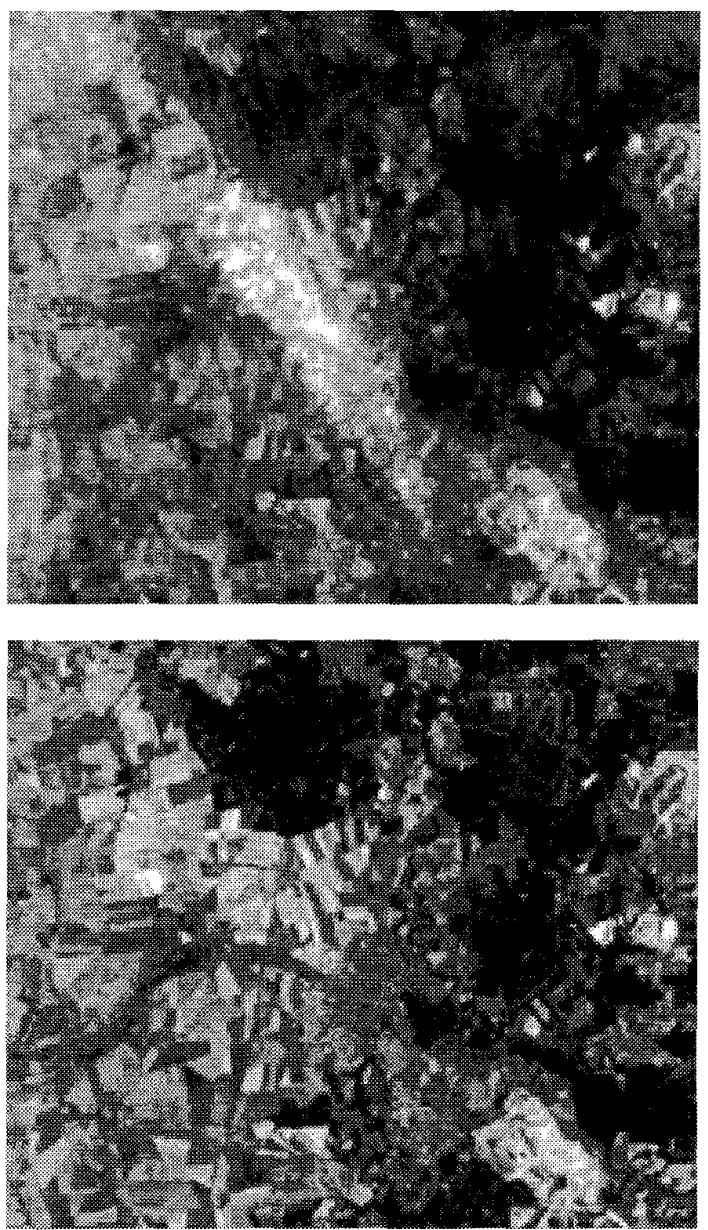

Figure 1 Band 1 original (top) and haze equalized (bottom)

As noted by Lavreau (1991), de-hazing can increase the correlation between bands. The eigenvalues summarize the spectral correlation structure of a multispectral image. Figure 3a compares the distribution of the eigenvalues corresponding to the three eigenvectors (principal components) computed from the visible bands before and after application of Lavreau's algorithm. A significant increase in the first eigenvalue indicates an increase in the correlation between the three visible bands. Figure $3 \mathrm{~b}$ compares the distribution of the eigenvalues before and after application of our haze equalization algorithm. The correlation structure of the haze equalized bands is more similar to the original (uncorrected) bands thus spectral balance is better preserved. 


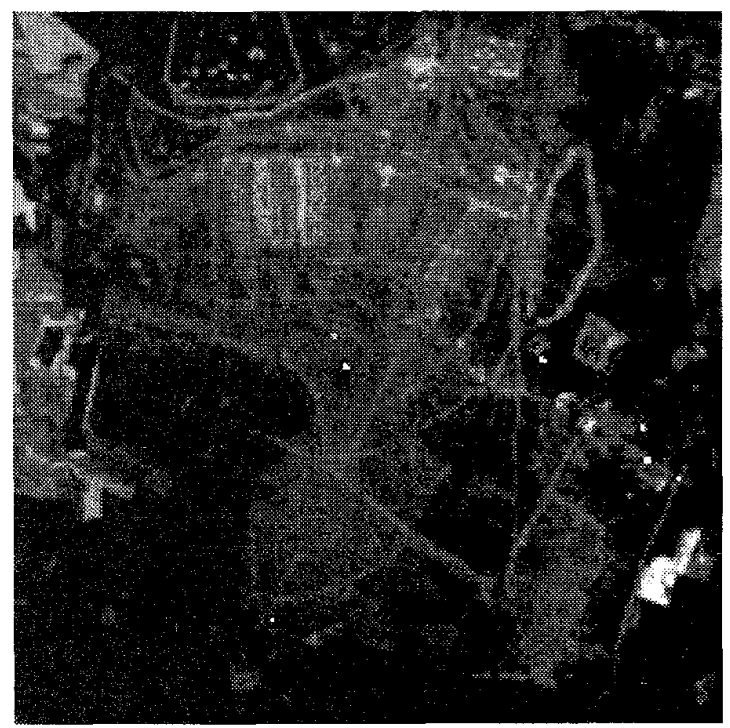

De-hazing algorithm

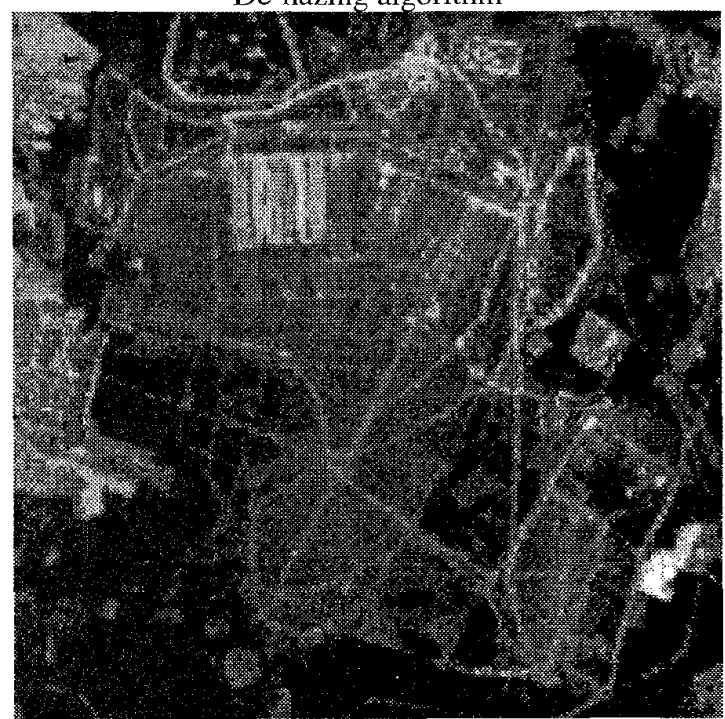

Haze-equalization algorithm

Figure 2 Visual comparison of algorithms

\section{CONCLUSION}

A new method for reducing the effects of space-varying, wavelength-dependent scattering in multispectral imagery caused by smoke and haze was described. The method converts an image in which space-varying scattering is present into an image where the scattering has, in effect, been equalized over the entire image so that previously developed techniques for removing constant scattering effects can be used. The algorithm predicts the values of those bands that are affected by scattering from those that are not on a pixel-by-pixel basis. It was compared to an earlier algorithm for removing space-varying haze and found to better preserve subtle detail in the image and spectral balance between bands. Future work will concentrate on testing the algorithm as part of a land cover classification system in order to assess its effect on classification accuracy.
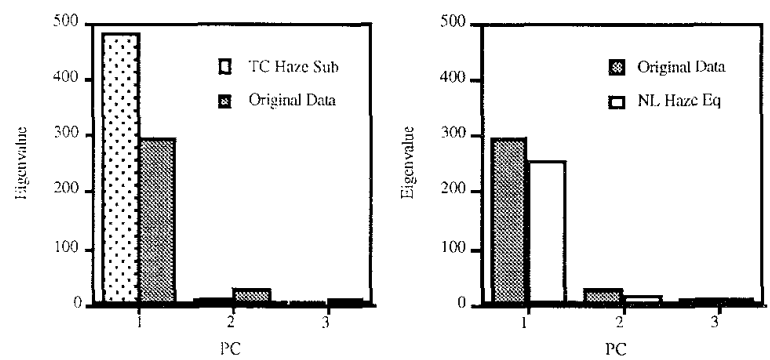

Figure 3 Eigenvalues before/after de-hazing (left) and hazeequalization (right)

\section{References}

[1] R.W. Sjoberg and B.K.P. Horn, "Atmospheric effects in satellite imaging of mountainous terrain," Applied Optics, Vol. 22, No. 11, pp 1702-1716, 1983.

[2] R.B. Crane, "Preprocessing techniques to reduce atmospheric and sensor variability in multispectral scanner data," Proceedings 7-th International Symposium on Remote Sensing of Environment, 1971.

[3] J.F. Potter and M.A. Mendlowitz, "On the determination of haze levels from Landsat data," Proceedings 10-th International Symposium on Remote Sensing of Environment, 1975.

[4] R.E. Crippen, "The regression intersection method of adjusting image data for band ratioing," Int. J. Remote Sensing, Vol. 8, No. 2, pp 137-155, 1987.

[5] J. Lavreau, "De-hazing Landsat Thematic Mapper images," Photogrammetric Engineering and Remote Sensing, Vol. 57, No. 10, pp 1297-1302, 1991.

[6] E.P. Crist and R.C. Cicone, "A physically-based transformation of thematic mapper data - The TM Tasseled Cap," IEEE Trans. Geoscience and Remote Sensing, Vol. 22, No. 3, May 1984.

[7] E. Schanda, Physical Fundamentals of Remote Sensing, Springer-Verlag, Berlin Heidelberg, 1986.

[8] A. Papoulis, Probability, Random Variables and Stochastic Processes, McGraw-Hill, 1965.

[9] E.P. Crist, R. Laurin and R.C.Cicone, "Vegetation and soils information contained in transformed Thematic Mapper data," Proceedings IGARSS, pp 14651470, 1986. 